\title{
Avifaunal study of Majalgaon Reservoir and their tributaries, District Beed, Marathwada region of Maharashtra, India
}

\author{
R. T. Pawar ${ }^{1 *}$, T.S. Pathan ${ }^{2}$, Y.M. Bhosale ${ }^{3}$ \\ ${ }^{1}$ Department of Zoology, Sunderrao Solanke Mahavidyalaya, Majalgaon Dist. Beed, (MH) India \\ ${ }^{2}$ Department of Zoology, Kalikadevi Arts, Commerce and Science College, Shirur Kasar Dist. Beed (MH) \\ ${ }^{3}$ Department of Zoology, Nanasaheb Yashwantrao Narayanrao Chavan ASC, College, Chalisgaon, Dist. Jalgaon (MH) \\ Correspondence Author: drtanvir7981@gmail.com, Mob. No.91+9028700713
}

Available online at: www.isroset.org

Received: 11/Jul/2019, Accepted: 20/Aug/2019, Online: 31/Aug/2019

\begin{abstract}
An avifaunal survey was conducted at Majalgaon Reservoir for two years, starting from July 2016 to June 2018. The study reveals a rich avian diversity a total of 84 species of birds belonging to 15 orders and 30 families were identified. As the water of the wetland is clear, rich in dissolved oxygen and supports variety of aquatic weeds and fishes, it has been found to be suitable for birds and attracts many migratory birds like large egert, purple heron, white bellied heron, asian open bill stork, greater flamingo, white naked stork etc. Continuous monitoring on the avifaunal diversity was suggested to evaluate the ecological status of the habitats and birds. It can be considered as an efficient tool to the environmentalists, policy makers in order to conserve such species, because, the role of birds in an ecosystem cannot be ignored.
\end{abstract}

Keywords : Majalgaon Reservoir, Avian diversity, Sindphana river, Migratory Birds etc.

\section{INTRODUCTION}

Bird community evaluation has become an important tool in biodiversity conservation and for identifying conservation actions in areas of high human pressure. Indian subcontinent is known for diverse and rich bird species whose taxonomy, distribution and their general habitat characteristics are well documented in India. Bird communities have been studied fairly well both in temperate and tropical forests. However, only a very little is known about bird community structure and their dynamics in India. Understanding the diversity and structure of bird communities is essential to delineate the importance of regional or local landscapes for avian conservation. Determinations of bird population in different habitats are central to understanding the community structure and niche relationships, as well as for intelligent management of populations. Moreover seasonal monitoring is equally important to trace the dynamic movement of birds in such habitats

Researcher have studied avian fauna in Marathwada as this area is blessed with various natural habitats such as river, reservoirs, hills, forests, grassland. (Yardi, et al., 2004) reported 64 species of birds in Salim Ali lake, Aurangabad. (Kulkarni, et al., 2005) reported 151 species of birds in and around Nanded city. Kulkarni et al., (2006) recorded 18 Piscivorous bird species in Dongarkheda irrigation tank. Dist. Hingoli. Kulkarni, et al., (2006) also recorded 93 species of birds in Shikhachiwadi Wadi, reservoir Dist. Nanded; Kulkarni et al., (2010) listed 62 bird species in forest Jaldhara, Kinwat. Dist.Nanded. (Balkhande et al., 2012) recorded 53 species of birds on river Godavari near Dhangar Takli; (Balkhande et al., 2012) recorded 50 species of birds near river Purna Dist. Parbhani.

Through this paper we would like to draw attention towards the least known avifauna of the Majalgaon reservoir and their tributaries, Beed district.

\section{MATERIALS AND METHODS}

\section{Study area}

The study area comes under the Marathwada region of Maharashtra (Fig. 1).

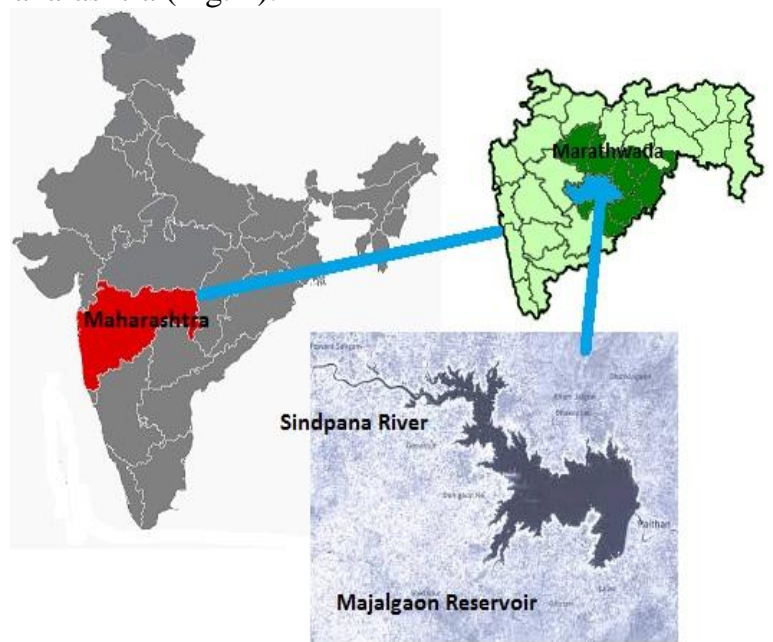

Fig. 1. Study area of Majalgaon Reservoir 
Field surveys were conducted for a period of two years starting from July 2016 up to June 2018 covering all the seasons. The areas were surveyed using binoculars and digital cameras for proper bird records. Direct observations and species noting was made by walking on channel of river, reservoir tracks, and reservoir areas. The observations were carried out at different points around the large wetland. Birds were identified following with the avifaunal field guidebooks (Kazmierczak et. al., 2003; Grimmett et. al., 2011) and Internet Birds database were used. The checklist of bird species is prepared by following the guidelines for checklist of birds (Abdulali, 1981; Ali and Replay, 1983). The population status observed of bird species was recorded as per ACOR rating (Buckland et. al., 1983). Current status of threatened categories was adopted from Bird Life International (2000).

\section{RESULTS AND DISCUSSION}

The present studies on bird community structure of Majalgaon Reservoir of Beed district, Marathwada region, Maharashtra revealed the presence of 84 species of birds belonging to 60 genera spread over 30 families and 15 orders. Further, it was interesting to note that the order fasseriformes dominated among the avian diversity with 29 species. (Table 1).

Family wise analysis showed that family Ardeidae (07 species) dominated the avifauna, followed by Anatidae, Columbidae, Turdinae (6 species each), Cuculidae (5 Species), Rallidae, Pycnonotidae (4 species each) Cionidae, Charadriidae, Pisttacidae, Alcedinidae, Hirundinidae, Paridae, Passerinae (3 species each), Phalacrocoracidae, Threskiornithidae, Phasianidae, Apodidae, Meropidae, Motacillidae, Laniidae, Sturnidae, Corvidae (2 species each) whereas, Podicipidae, Phoenicopteridae, Gruidae, Recurviorstridae, Strigidae, Estrildidae and Dicruridae (1 species each) were poorly represented in the area (Table 1). Similarly, Manakadan and Pittie (2000) have reported Muscicapidae as the largest bird family of India with 370 species. Recently, Mahabal (2000) reported maximum number of birds (105 species) under this family. Similarly, Thakur (2008) also found Muscicapidae as the biggest family of birds with 67 species from Himachal Pradesh.

Analysis of data on residential status revealed that out of 84 species, 33 were resident common and rest 51 showed migrant common, residential migrant common, uncommon rare, migrant rare, resident rare, uncommon, seasonally migrant common, uncommon, rare and breeding migrant rare, uncommon (Table 1). The present work is in conformity with the earlier work of S. P. Chavan et.al. (2015) was reported 168 birds from Godaveri river basin Nanded, Thakur et al. (2003) carried out in Balh Valley of Mandi district in lower Himalayan region of Himachal Pradesh. Similarly, this investigation is in agreement with the earlier works of Mahabal and Mukherjee (1991), Thakur et al. (2002), Mahabal (2000), Mattu and Thakur (2006), Thakur (2008) and Thakur et al. (2010) who also reported resident, altitudinal migrant, summer and winter visitor birds in different areas of Himachal Pradesh.

Analysis of data as per ACOR and IUCN status the ACOR rating only 12 species were abundant, 33 common, 23 occasional and 16 species were rare. According to IUCN categorization 79 species were least concern, 03 nearly threatened and 01 species from vulnerable and critically endangered. No any bird species from threatened and endangered (EN) category was sighted at any selected bird habitat. Similarly, there is no report of any species of bird which has been extinct from this region in recent time. (Table 1) The present work is in conformity with the earlier work according to Kulkarni et. al. (2005) had extensively studied the birds in and around Nanded city and enlisted with their categorization and ACOR rating for abundance. S. P. Chavan et.al. (2015) also reported ACOR categorization and IUCN status of birds from Godaveri river basin Nanded.

\section{ACKNOWLEDGEMENTS}

The authors are highly acknowledged to the Principal, Sunderrao Solanke Mahavidyalaya, Majalgaon for providing the necessary facilities to carry out the study.

\section{REFERENCES}

[1]. Abdulali H. (1981). Checklist of Birds of Maharashtra, BNHS, Mumbai, PP: 1-35.

[2]. Ali, S. and Ripley, S.D. (1983a). Handbook of the Birds of India and Pakistan (compact edition). Oxford University Press, New Delhi. PP. 737

[3]. Balkhande J. V., Bhowte C. S., Kulkarni AN (2012). Checklist of Birds of River Godavari, Dhangartakli near Purna, Dist. Parbhani, Maharashtra, Bionano Frontier 5(2).

[4]. BirdLife International (2001a). Threatened Birds of Asia: The BirdLife International Red Data Book-Vol. 1. Birdlife International. Cambridge, UK, 1516pp.

[5]. Buckland S. T., Anderson D. R., Burnham K. P., Laake J. L. (1993). Distance sampling: Estimating abundance of biological populations, Chapman and Hall, London, P. 446.

[6]. Grimmett R., Inskipp C., Inskipp T. (2011). Birds of the Indian Subcontinent. 2nd ed. New Delhi: Oxford University Press.

[7]. Kazmierczak, K., 2000. A field Guide to the Birds of India, Sri Lanka, Pakistan, Nepal Bhutan, Bangladesh and the Maldives. Om Book Service, New Delhi.

[8]. Kulkarni A. N., Bhowte C. S., Kanwate V. S. (2006 a). Bird census in Nanded region (Maharashtra). Bioinfolet 3 (3), 173-178.

[9]. Kulkarni A. N., Kanwate V. S. (2006 c). Piscivorous birds of Dongarkheda irrigation tank. Dist Hingoli, Maharashtra. Jr. Of Aqua. Biol. 21 (1), 86-87.

[10]. Kulkarni A. N., Kanwate V. S. (2010). Avian fauna of forest Jaldhara, Kinwat Dist. Nanded, Maharashtra. Jr. of Aqua. Biol. 25 (1), 46-51.

[11]. Kulkarni A. N., Kanwate V. S., Deshpande V. D. (2005). Birds in and around Nanded city, Maharashtra. Zoo s Print Journal 20 (11), 2076-2078. 
[12]. Kulkarni A. N., Kanwate V. S., Deshpande V. D. (2006 b). Checklist of Birds of Shikhachiwadi Reservoir. District Nanded, Maharashtra. J. Of Aqua. Biol 21(10), 80-85.

[13]. Mahabal, A. (2000). Birds of Talra Wildlife Sanctuary in lower Western Himalaya, Himachal Pradesh, with notes on their status and altitudinal movements. Zoos' Print Journal, 15 (10): 334-338.

[14]. Mahabal,A. and Mukherjee, R. (1991). Birds of Mandi District (Himachal Pradesh). Newsletter for Birdwatchers 31 (1\&2): 8-9.

[15]. Manakadan, R. and Pittie, A. (2000). Standardised common and scientific names of the birds of the Indian subcontinent. Buceros 6 (1): $1-37$

[16]. Shivaji P. Chavan, Dilip Dudhmal, Shrikrishna Hambarde and A. N. Kulkarni (2015). Birds from godavari river basin in Nanded district of Maharashtra State, India: Annotated status and new reports, Int. J. Curr. Res. Aca. Rev.: 3 (4), 328-351.

[17]. Thakur, M.L. (2008). Studies on status and diversity of avifauna in Himachal Pradesh. Ph.D. thesis, Himachal Pradesh University,
Shimla, India. PP 306.

[18]. Thakur, M.L., Mattu, V.K., Hira Lal, Sharma, V., Hem Raj and Thakur, V. (2010). Avifauna of Arki Hills, Solan (Himachal Pradesh), India. Indian Birds 5 (6): 162-166.

[19]. Thakur, M.L.; Mattu, V.K. and Sharma, R.M. (2006). Bird diversity and status in Tara Devi, Shimla, Himachal Pradesh. In: Biodiversity and Environment (Eds.: Pandey B.N. and Kulkarni G.K.). A.P.H. Pub., New Delhi.

[20]. Thakur, M.L.; Paliwal, R.; Tak, P.C. and Mattu, V.K. 2003. Birds of Balh Valley, District Mandi, Himachal Pradesh, India. Annals of Forestry 11 (1): 113-126.

[21]. Thakur, M.L.; Paliwal, R.; Tak, P.C.; Mehta, H.S. and Mattu, V.K. (2002). Birds of Kalatop- Khajjiar Wildlife Sanctuary, Chamba (H.P.). Cheetal 41 (3 \& 4): 29-36.

[22]. Yardi D, Patil SS, Auti RG. 2004. Diversity of Avian Fauna from Salim Ali Lake of Aurangabad. 21st Meet of Birds Lovers of Maharashtra, Nanded, 3-4 April 2004

Table 1: Systematic list of birds of Majalgaon Reservoir, Marathwada region, Maharashtra

\begin{tabular}{|c|c|c|c|c|}
\hline $\begin{array}{l}\text { Sr. } \\
\text { No. }\end{array}$ & Taxon & Common Name & $\begin{array}{l}\text { Population, } \\
\text { IUCN Status }\end{array}$ & Ecological status \\
\hline & Family I: Podicipidae & & & \\
\hline \multirow{2}{*}{1} & Order II: Pelecaniformes & & & \\
\hline & Family II : Phalacrocoracidae & & & \\
\hline 2 & Phalacrocorax niger (Vieillot, 1817) & Little Cormorant & $\mathrm{A}(\mathrm{LC})$ & RMC \\
\hline 3 & Family III: Ardeidae & & & \\
\hline 4 & Ardeola grayii (Skyes, 1832) & Indian pond Heron & A (LC) & $\mathrm{RC}$ \\
\hline 5 & Egretta garzetta (Linnaeus, 1766) & Little Egret & $\mathrm{C}(\mathrm{LC})$ & $\mathrm{RM}$ \\
\hline 6 & Bubulcus ibis (Linnaeus, 1758) & Cattle Egret & A (LC) & $\mathrm{RM}$ \\
\hline 7 & Casmerodius albus (Linnaeus, 1758) & Large Egert & $\mathrm{O}(\mathrm{LC})$ & $\mathrm{Mr}$ \\
\hline 11 & Mycteria leucocephala (Pennant, 1769) & Painted Stork & $\mathrm{C}(\mathrm{NT})$ & $\mathrm{MC}$ \\
\hline 12 & Anastomus oscitans(Boddaert, 1783) & Asian Open bill Stork & $\mathrm{R}(\mathrm{LC})$ & $\mathrm{Mr}$ \\
\hline \multirow[t]{2}{*}{13} & Ciconia episcopus (Boddaert, 1783) & $\begin{array}{l}\text { White Necked/Wooly } \\
\text { Stork }\end{array}$ & $\mathrm{O}(\mathrm{LC})$ & $\mathrm{WMr}$ \\
\hline & Family V: Threskiornithidae & & & \\
\hline 14 & Pseudibis papillosa (Temminck, 1824) & $\begin{array}{l}\text { Oriental White/Black } \\
\text { Head Ibis }\end{array}$ & $\mathrm{R}(\mathrm{LC})$ & MU \\
\hline \multirow[t]{3}{*}{15} & Platalea leucorodia (Linnaeus, 1758) & Euresion Spoonbill & $\mathrm{C}(\mathrm{LC})$ & WMU \\
\hline & Order IV: Phoenicopteriformes & & & \\
\hline & Family VI: Phoenicopteridae & & & \\
\hline \multirow[t]{2}{*}{16} & Phoenicopterus ruber (Linnaeus, 1758) & Greater flamingo & $\mathrm{R}(\mathrm{LC})$ & WMr \\
\hline & Order V: Ansariformes & & & \\
\hline \multirow{2}{*}{22} & Order VI: Galliformes & & & \\
\hline & Family VIII: Phasianidae & & & \\
\hline 23 & Pavo cristatus (Linnaeus, 1758) & Indian Peafowl & A (LC) & $\mathrm{RC}$ \\
\hline
\end{tabular}




\begin{tabular}{|c|c|c|c|c|}
\hline 24 & Coturnix Coturnix (Linnaeus, 1758) & Common Quail & A (LC) & $\mathrm{RC}$ \\
\hline & Order VII: Gruiformes & & & \\
\hline & Family IX: Gruidae & & & \\
\hline \multirow{2}{*}{25} & Grus antigone (Linneaus, 1758) & Sarus Crane & $\mathrm{O}(\mathrm{VU})$ & RU \\
\hline & Family X: Rallidae & & & \\
\hline 26 & Amaurornis phoenicurus (Pennant, 1769) & White-breasted Waterhen & A (LC) & $\mathrm{RC}$ \\
\hline 27 & Porphyrio porphyrio (Linnaeus, 1758) & Purple Moorhen & A (LC) & $\mathrm{RC}$ \\
\hline 28 & Fulica atra (Linnaeus, 1758) & Eurasian Coot & $\mathrm{O}(\mathrm{LC})$ & $\mathrm{RC}$ \\
\hline \multirow[t]{3}{*}{29} & Gallinula chloropus (Linnaeus, 1758) & Common Moorhen & C (LC) & $\mathrm{RC}$ \\
\hline & Order VIII: Charadriiformes & & & \\
\hline & Family XI: Charadriidae & & & \\
\hline 30 & Vanellus duvaucelii (Lesson, 1826) & River Lapwing & R (NT) & $\mathrm{Rr}$ \\
\hline 31 & Vanellus indicus (Boddaert, 1783) & Red-wattled Lapwing & $\mathrm{C}(\mathrm{LC})$ & $\mathrm{RC}$ \\
\hline \multirow{2}{*}{32} & Vanellus malabricus (Boddaert, 1783) & Yellow wattled Lawping & $\mathrm{O}(\mathrm{LC})$ & RU \\
\hline & Family XII: Recurvirostridae & & & \\
\hline \multirow[t]{3}{*}{33} & Himantopus himantopus (Linnaeus, 1758) & Black winged stilt & A (LC) & WMC \\
\hline & Order IX: Columbiformes & & & \\
\hline & Family XIII: Columbidae & & & \\
\hline 34 & Columba livia (Gmelin, 1789) & Blue Rock Pigeon & C (LC) & $\mathrm{RC}$ \\
\hline 35 & Streptopelia senegalensis (Linnaeus, 1766) & Little Brown Dove & $\mathrm{C}(\mathrm{LC})$ & RU \\
\hline 36 & Streptopelia chinensis (Scopoli, 1786) & Spotted Dove & $\mathrm{O}(\mathrm{LC})$ & RU \\
\hline 37 & Streptopelia decaocto (Frivaldszky, 1838) & Eurasian Collared-Dove & $\mathrm{C}(\mathrm{LC})$ & $\mathrm{RC}$ \\
\hline 38 & Streptopelia tranquebarica (Hermann, 1804) & Red collared Dove & $\mathrm{R}(\mathrm{LC})$ & RMC \\
\hline \multirow[t]{3}{*}{39} & Treron phoenicoptera (Latham, 1790) & $\begin{array}{l}\text { Yellow-legged Green- } \\
\text { Pigeon }\end{array}$ & $\mathrm{R}(\mathrm{LC})$ & $\mathrm{Rr}$ \\
\hline & Order X: Psittaciformes & & & \\
\hline & Family XIV: Psittacidae & & & \\
\hline 40 & Psittacula eupatria (Linnaeus, 1766) & Alexandrine Parakeet & $\mathrm{O}(\mathrm{LC})$ & $\mathrm{RC}$ \\
\hline 41 & Psittacula krameri (Scopoli, 1769) & Rose-ringed Parakeet & $\mathrm{O}(\mathrm{LC})$ & $\mathrm{RC}$ \\
\hline \multirow[t]{3}{*}{42} & Psittacula roseate (Biswas, 1951) & Blssom headed Parakeet & $\mathrm{R}(\mathrm{NT})$ & $\mathrm{RC}$ \\
\hline & Order XI : Cuculiformes & & & \\
\hline & Family XV: Cuculidae & & & \\
\hline 43 & Clamator jacobinus (Boddaert, 1783) & Pied Crested Cuckoo & $\mathrm{O}(\mathrm{LC})$ & $\mathrm{BMr}$ \\
\hline 44 & Hierococcyx varius (Vahl, 1797) & Brainfever Bird & C (LC) & BMU \\
\hline 45 & Cuculus micropterus (Gould, 1838) & Indian Cuckoo & A (LC) & $\mathrm{RC}$ \\
\hline 46 & Cuculus canorus (Linnaeus, 1758) & Common Cuckoo & $\mathrm{R}(\mathrm{LC})$ & $\mathrm{RC}$ \\
\hline \multirow[t]{3}{*}{47} & Eudynamys scolopacea (Linnaeus, 1758) & Asian Koel & $\mathrm{C}(\mathrm{LC})$ & $\mathrm{RC}$ \\
\hline & Order XII: Strigiformes & & & \\
\hline & Family XVI: Strigidae & & & \\
\hline \multirow[t]{3}{*}{48} & Athene brama (Temminck, 1821) & Spotted Owlet & C (LC) & $\mathrm{RC}$ \\
\hline & Order XIII: Apodiformes & & & \\
\hline & Family XVII: Apodidae & & & \\
\hline 49 & Apus affinis (J.E. Gray, 1830) & House Swift & A (LC) & $\mathrm{RMr}$ \\
\hline \multirow[t]{3}{*}{50} & Apus apus (Linneaus 1758) & Common swift & C (LC) & $\mathrm{Rr}$ \\
\hline & Order XIV: Coraciiformes & & & \\
\hline & Family XVIII: Alcedinidae & & & \\
\hline 51 & Alcedo atthis (Linnaeus, 1758) & Small Blue Kingfisher & $\mathrm{C}(\mathrm{LC})$ & RU \\
\hline 52 & Halcyon smyrnensis (Linnaeus, 1758) & White-breasted Kingfisher & $\mathrm{C}(\mathrm{LC})$ & RU \\
\hline \multirow[t]{2}{*}{53} & Ceryle rudis (Linnaeus, 1758) & Pied Kingfihser & $\mathrm{C}(\mathrm{LC})$ & $\mathrm{RU}$ \\
\hline & Family XIX: Meropidae & & & \\
\hline 54 & Merops orientalis (Latham, 1801) & Small Bee-eater & C (LC) & RMC \\
\hline \multirow[t]{3}{*}{55} & Merops philippines (Linnaeus, 1766) & Blue tailed bee eater & $\mathrm{C}(\mathrm{LC})$ & RMU \\
\hline & Order XV: Passeriformes & & & \\
\hline & Family XX: Hirundinidae & & & \\
\hline 56 & Hirundo rustica (Linnaeus, 1758) & Common Swallow & $\mathrm{O}(\mathrm{LC})$ & RMC \\
\hline 57 & Hirundo daurica (Linnaeus, 1771) & Red-rumped Swallow & $\mathrm{O}(\mathrm{LC})$ & RMC \\
\hline \multirow[t]{2}{*}{58} & Hirundo smithii (Leach, 1818) & Coire tailed swallow & $\mathrm{O}(\mathrm{LC})$ & $\mathrm{WMr}$ \\
\hline & Family XXI: Motacillidae & & & \\
\hline 59 & Motacilla alba (Linnaeus, 1758) & White Wagtail & $\mathrm{R}(\mathrm{LC})$ & MU \\
\hline
\end{tabular}




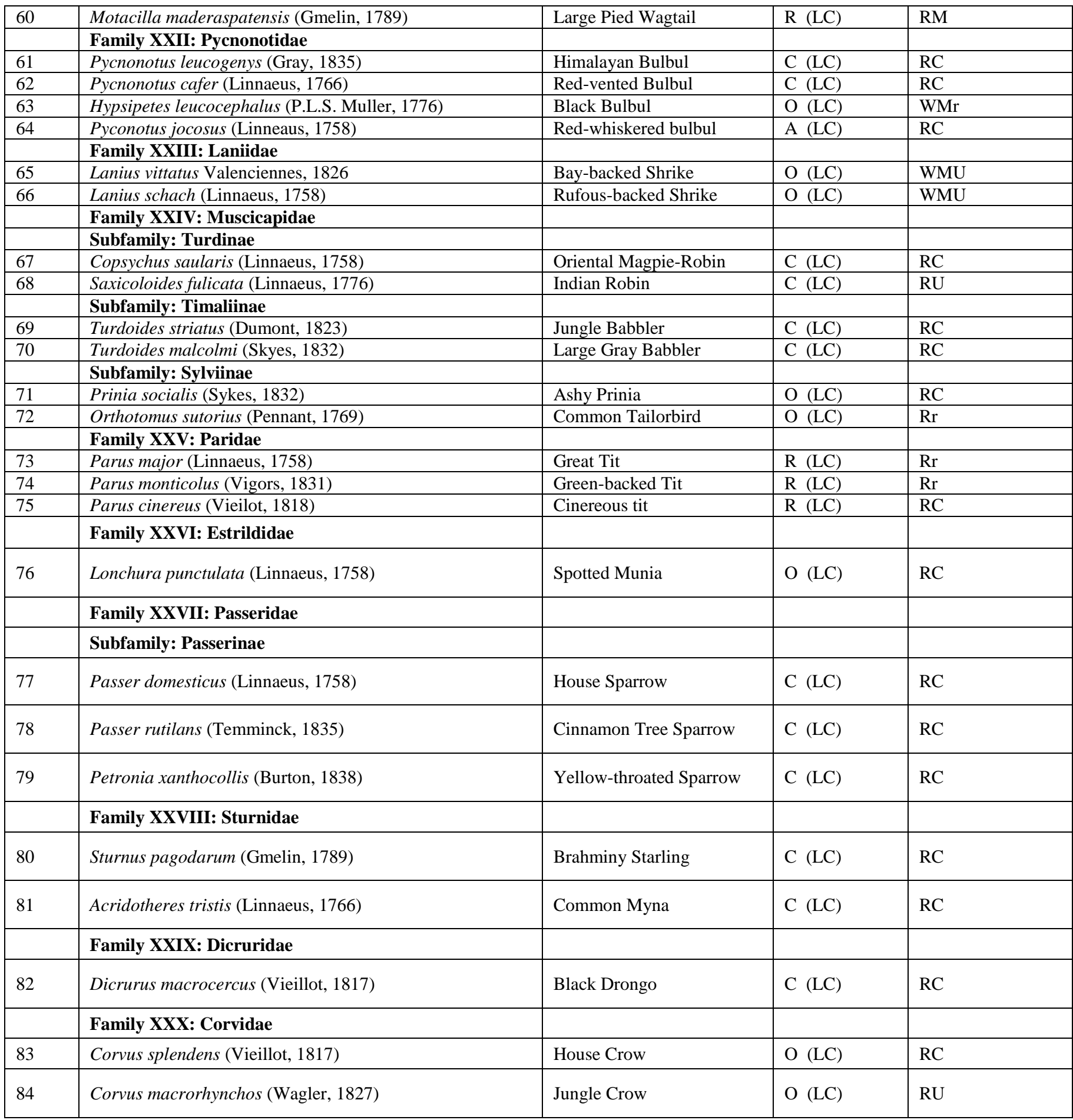

Abbreviation in ACOR are $\mathrm{A}=$ Abundant, $\mathrm{C}=$ Common, $\mathrm{O}=$ Occasional, $\mathrm{R}=$ Rare

Abbreviation used for Ecological rating and status are $\mathrm{WM}=$ Winter Migrant, $\mathrm{WMr}=\mathrm{Winter}$ Migrant Rare, WMU $=\mathrm{Winter}$ Migrant Uncommon, WMC $=$ Winter Migrant Common, RU = Resident Uncommon, $\mathrm{Rr}=\mathrm{Resident}$ Rare, $\mathrm{RMR}=\mathrm{Residence}$ Migrant Rare, $\mathrm{RMC}=$ Residential Migrant Common, $\mathrm{RMC}=$ Residential Migrant Uncommon, $\mathrm{RC}=\mathrm{Resident} \mathrm{Common}, \mathrm{BM}=$ Breeding Migrant, $\mathrm{BMR}=$ Breeding Migrant Rare, $\mathrm{BMU}=$ Breeding Migrant Uncommon, $\mathrm{PM}=$ Passage Migrant.

Abbreviation for IUCN Status are LC $=$ Least Concern, NT $=$ Nearly Threatened, T $=$ Threatened, VU $=$ Vulnerable, EN $=$ Endangered, $\mathrm{CR}=$ Critically Endangered . 